\title{
Reprodutibilidade da curva força-tempo do estilo "Crawl" em protocolo de curta duração
}

CDD. 20.ed. 796.023

797.2
Augusto Carvalho BARBOSA*

Rodrigo Maciel ANDRADE ${ }^{* *}$ Alexandre MOREIRA ${ }^{* *}$ Júlio Cerca SERRÃO** Orival ANDRIES J ÚNIOR*
*Faculdade de Educação Física, Universidade Estadual de Campinas.

**Escola de Educação Física e Esporte, Universidade de São Paulo.

\section{Resumo}

0 objetivo deste estudo foi analisar a reprodutibilidade dos parâmetros biomecânicos da curva força-tempo do estilo "Crawl" em um protocolo de $10 \mathrm{~s}$ no nado atado. Dezesseis nadadores do sexo masculino (idade: $20,4 \pm 4,0$ anos; tempo na prova de $100 \mathrm{~m}$ livre: $53,68 \pm 0,99 \mathrm{~s}$ ) realizaram dois esforços máximos de 10 s no nado atado. Os parâmetros força pico, força média, taxa de desenvolvimento de força, impulso, duração da braçada, tempo para atingir a força pico e força mínima foram representados pela média de oito braçadas consecutivas obtidas em cada tentativa. Utilizou-se o teste $t$ para observar as diferenças entre os esforços para cada parâmetro. 0 nivel de significância estabelecido foi de $5 \%$. A reprodutibilidade relativa foi medida pelo coeficiente de correlação de Pearson e a consistência entre as duas tentativas pelo coeficiente de correlação intraclasse (CCI). A reprodutibilidade absoluta foi verificada pelo coeficiente de variação (CV). Não foi demonstrada diferença estatisticamente significante para nenhum parâmetro biomecânico quando comparados os dois esforços. Os elevados $\mathrm{CCl}$ e baixos $\mathrm{CV}$ indicaram alta consistência interna dos parâmetros analisados. Conclui-se que os parâmetros biomecânicos analisados a partir do nado atado são reprodutíveis quando empregado protocolo de curta duração o que demonstra a possibilidade de utilização do protocolo com alto grau de confiabilidade, por parte de treinadores e atletas.

UnITERMOS: Reprodutibilidade; Natação; Força propulsora; Nado atado.

\section{Introdução}

O nado completamente atado é apontado como o teste mais utilizado na mensuração da força propulsora na natação (Dopsaj, MATKOVIC \& ZDRAVKOVIC, 2000; Pessôa-Filho, Nascimento \& Denadai, 2008) e é assim designado pelo fato de o nadador executar as ações características da modalidade conectado a um transdutor de força por um cabo inextensível (Castro, Oliveira, Moré \& Mota, 2010).

Embora a condição de velocidade nula do nado atado possa alterar a duração, trajetória e velocidade dos segmentos propulsores (Maglischo, MaglisChO, Sharp, Zier \& Katz, 1984), e fazer com que o avaliado não sofra ação do arrasto hidrodinâmico (BARbosa, Dopsaj, Okicic \& Andries Júnior, 2010), estudos têm sinalizado para a existência de fatores que sustentam sua validade ecológica, como a similaridade de respostas metabólicas e eletromiográficas, quando comparado ao nado convencional (Bollens, Annemans, Vaes \& Clarys, 1988; Bonen, Wilson, Yarkony \& Belcastro, 1980; Cabri, Annemans, Clarys, Bollens \& Publie, 1988; HolMÉR, 1979) e também sua a sensibilidade quanto à identificação e monitoramento das adaptaçôes induzidas pelo treinamento (Papoti, Martins, Cunha, Zagatto \& Gobatto, 2007).

Os parâmetros biomecânicos comumente empregados na análise da força propulsora são o impulso, a força média e/ou a força pico, pois apresentam relação significante com a velocidade de nado (Adams, Martin, Yeater \& Gilson, 1983; BARbosa et al., 2010; CASTRO et al., 2010; Dopsaj, MatKovic \&ZDraVKOVIC, 2000; Morouço, KeSKInEN, 
Vilas-Boas \& Fernandes, 2011; Papoti, Martins, CunHa, ZagatTo \& Gobatto, 2003; Risch \& CASTRO, 2007; Szmuchrowski, Ferreira, Carvalho \& Ferreira, 2007; Yeater, Martin, White, Gilson, 1981) e, por isso, são considerados em diferentes modelos de predição de desempenho (BARBOSA et al., 2010; CASTRO et al., 2010; DopsaJ, MatKOVIC \& ZDRAVKOVIC, 2000; PAPOTI et al., 2003). A magnitude de expressão desses parâmetros biomecânicos se relaciona com fatores como o estilo do nado (YEATER et al., 1981), o gênero (ADAMS et al., 1983; MARINHO \& GoMES, 1999), a frequência de braçadas (ADAMS et al., 1983; CABRI et al., 1988), o nível de qualificação do nadador (MARINHO, 2002) e a especialidade competitiva (ADAMS et al., 1983; Marinho, Bernhoeft, Moura, Cipolli \& Andries JúNIOR, 2005; MorouçO et al., 2011).

No contexto esportivo, a utilização de procedimentos de avaliação e de monitoramento através de testes e medidas tem como objetivo principal controlar as possíveis alterações de rendimento ao longo de uma temporada e/ou em momentos pontuais do processo de treinamento (Moreira, Maia, Lizana, Martins \& Oliveira, 2008). Para isso, é necessário garantir que uma dada medida que faça parte de uma pesquisa ou sustente o controle do rendimento de um atleta seja válida, específica e reprodutível (AtKinson \& Nevill, 1998). A reprodutibilidade pode ser definida como a consistência de medidas ou

\section{Metodologia}

\section{Amostra}

Dezesseis nadadores homens fizeram parte do presente estudo (média e desvio-padrão para idade, estatura, envergadura, massa corporal e tempo de treinamento de 20,4 $\pm 4,0$ anos, $1,84 \pm 0,07 \mathrm{~m}, 1,89$ $\pm 0,08 \mathrm{~m} \mathrm{e} 77,8 \pm 9,1 \mathrm{~kg}, 10,2 \pm 4,6$ anos, respectivamente). O nível competitivo dos mesmos foi determinado considerando os índices mínimos adotados pelas entidades responsáveis para a participação na prova de 100 m livre no Pan-Pacífico (49,23 s), Campeonato Brasileiro Sênior (54,50 s) e o Campeonato Paulista de Verão $(56,70 \mathrm{~s})$, considerados os principais eventos da categoria Sênior em nível internacional, nacional e estadual, respectivamente, em piscinas de $50 \mathrm{~m}$ no ano de 2010. Nesta prova, os nadadores investigados no presente estudo obtiveram um tempo médio de $53,68 \pm 0,99 \mathrm{~s}$ e foram, portanto, caracterizados como sendo de nível nacional. de um desempenho individual em um teste (ATKINSON \& NeVILL, 1998) e o conhecimento desta faz-se fundamental para que o monitoramento possa ser realizado e interpretado apropriadamente.

Neste sentido, apesar da ampla utilização do nado atado para avaliação e monitoramento do processo de treinamento na natação, o conhecimento da confiabilidade e reprodutibilidade das medidas são ainda limitados, sobretudo quando se considera as diferentes durações dos protocolos utilizados (que variam de 5 a 60 s).

Assim, considerando que em esforços máximos essa distinção diferenciará as demandas metabólicas e neuromusculares (DOPSAJ, MATKOVIC, ThANOPOULOS $\&$ OKICIC, 2003; PAPOTI et al., 2003), e que os estudos prévios sobre reprodutibilidade em protocolos de curta duração se restringiram apenas à análise da força pico (Marinho \& ANDries Júnior, 2001), o presente estudo teve por objetivo analisar a reprodutibilidade dos parâmetros biomecânicos da curva força-tempo do estilo "Crawl" em um protocolo de $10 \mathrm{~s}$ no nado atado. $\mathrm{O}$ conhecimento da reprodutibilidade de diferentes parâmetros biomecânicos analisados a partir deste protocolo é fundamental para as investigações que utilizam o referido teste, assim como para treinadores e atletas que o fazem regularmente com o objetivo de monitorar as repostas decorrentes do processo de preparação.

Os atletas estavam amplamente familiarizados com o teste, já que realizavam o procedimento frequentemente durante as sessôes de treino com o objetivo de monitorar as respostas agudas e crônicas do treinamento. Os atletas preencheram o termo de consentimento livre e esclarecido, após receberem todas as informações a respeito dos objetivos e procedimentos do estudo. O projeto foi aprovado pelo Comitê de Ética em Pesquisa da Universidade onde o estudo foi realizado (992/2008).

\section{Procedimentos}

Os procedimentos de teste ocorreram ao final da etapa de competição, em apenas um dia, no horário habitual de treino da equipe (entre 9:00 e 11:00 da manhã). O teste foi realizado 24 horas após a última sessão de treinamento dos atletas, que foram instruídos a manter suas dietas habituais. Após um aquecimento 
padronizado (10 minutos de alongamento ativo fora da água + 10 minutos de nado submáximo + quatro repetições de $15 \mathrm{~m}$ em máxima velocidade com intervalo de um minuto e trinta segundos $+100 \mathrm{~m}$ de nado solto), os nadadores realizaram dois esforços máximos de 10 s no nado atado utilizando o estilo "Crawl". O intervalo de recuperação entre as tentativas (esforços) foi de quatro minutos, a fim de garantir a ressíntese do sistema ATP-CP (GLAISTER, 2005). O início e o término do teste foram determinados por sinal sonoro (apito) após aproximadamente cinco segundos de nado moderado. No intuito de minimizar os efeitos da transição da intensidade do nado foi dado um intervalo de um segundo entre o apito e o início da aquisição dos dados. Durante o período de aquisição dos dados, os nadadores bloquearam a respiração. $\mathrm{O}$ batimento de pernas foi permitido e a frequência de braçada foi escolhida arbitrariamente pelos atletas.

O sistema (CEFISE, Nova Odessa, Brasil), ilustrado na FIGURA 1, utilizou uma célula de carga do tipo "Strain Gage", isolada contra umidade, com capacidade de $2000 \mathrm{~N}$ na condição de tração e/ou compressão e precisão aproximada de $30 \mathrm{~g}$. Uma de suas extremidades foi fixada ao bloco de partida por intermédio de um suporte de alumínio a uma distância de aproximadamente três centímetros da linha da água, enquanto a outra se conectou a um sistema de cabos por onde o nadador foi atado.

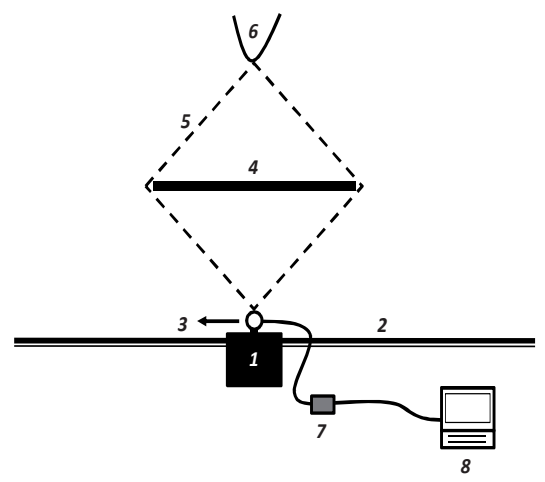

1 - bloco de partida 2 - parede da piscina; 3 - célula de carga;

4 - barra flutuante;

5 - cabos inextensíveis;

6 - cinturão;

7 - interface

8 - computador

FIGURA 1 - Vista superior do nado atado.

Os sinais da deformação mecânica da célula de carga, gerados a partir dos esforços do nadador e adquiridos a uma frequência de $600 \mathrm{~Hz}$, foram suavizados pelo filtro "butterworth" de quarta ordem (PAPOTI et al., 2003) com uma frequência de corte de oito hertz, definida pelo processo de análise residual proposto por WINTER (1990). A FIGURA 2 mostra uma curva típica do nado "crawl” em 10 s.

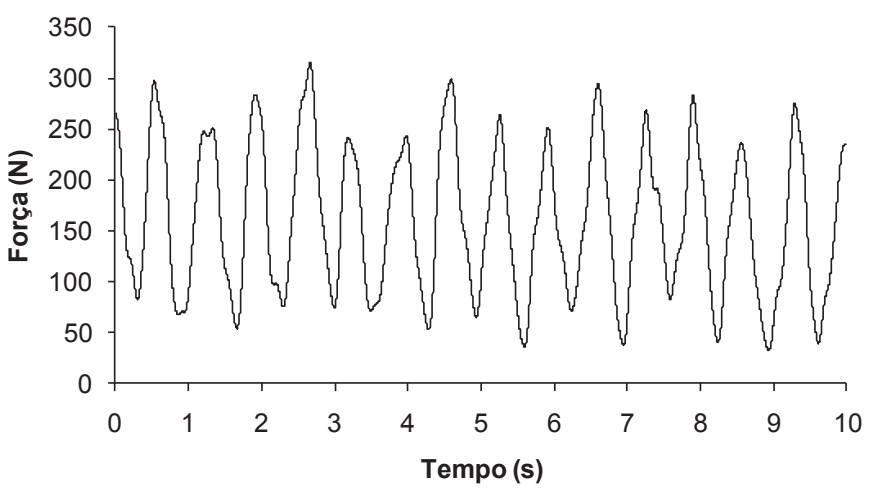

FIGURA 2 - Exemplo de uma curva força-tempo típica obtida após filtragem no teste de 10 s em nado atado.

Previamente às coletas o sistema foi calibrado utilizando incrementos de $20 \mathrm{~kg}$, até a carga máxima de $100 \mathrm{~kg}$. Assim, o sinal digital obtido pôde ser convertido em unidades de força $(\mathrm{N})$ através da equação de regressão gerada. Os resultados da ANOVA mostraram que o modelo de regressão linear foi estatisticamente significante $(F=150401,61, p<0,0001)$, apresentou um erro típico de medida de $2,16 \mathrm{~N}$, e altos coeficientes de correlação $(r=1,00)$ e determinação $\left(r^{2}=1,00\right)$, evidenciando nível de associação perfeito entre as variáveis.

Conforme procedimento descrito na literatura (Dopsaj, Matkovic \& Zdravkovic, 2000; Dopsaj et 
$F_{\text {pico }}=$ força pico;

$\mathrm{I}_{\mathrm{mp}}^{\mathrm{pico}} \mathrm{F}=$ impulso, representado pela área da curva;

$\mathrm{F}_{\min 1}=$ início da braçada;

$F_{\min 2}^{\min }=$ fim da braçada;

$\Delta \mathrm{t}=$ variação do tempo; $\Delta \mathrm{F}=$ variação da força. al., 2003), cada braçada foi analisada isoladamente. Para cada tentativa foram consideradas oito braçadas consecutivas e em cada uma delas foram obtidos seguintes parâmetros biomecânicos (exemplificados na FIGURA 3):

1. Força Pico $\left(\mathrm{F}_{\text {pico }}\right)$ : maior valor de força encontrado na braçada, expresso em $\mathrm{N}$.

2. Força Média $\left(\mathrm{F}_{\text {méd }}\right)$ : média da força gerada durante toda a braçada, expressa em $\mathrm{N}$.

3. Força Mínima $\left(\mathrm{F}_{\text {mín }}\right)$ : menor valor de força encontrado na braçada, expressa em N. Um mesmo valor de força mínima define o início de uma braçada e o término da braçada anterior.
4. Duração da braçada (DUR): tempo decorrido entre dois instantes de $\mathrm{F}_{\text {mín }}\left(\mathrm{F}_{\text {mín } 1}\right.$ e $\mathrm{F}_{\text {mín } 2}, \mathrm{~F}_{\text {mín } 2}$ e $\mathrm{F}_{\text {mín } 3} \ldots$ $\mathrm{F}_{\text {mín8 }}$ e $\mathrm{F}_{\text {mín } 9}$ ), expresso em milésimos de segundo.

5. Tempo para atingir a força pico $\left(\mathrm{TF}_{\text {pico }}\right)$ : intervalo de tempo decorrido entre $\mathrm{F}_{\text {mín }}$ e $\mathrm{F}_{\text {pico }}$, expresso em milésimos de segundo

6. Taxa de desenvolvimento de força (TDF): razão entre a variação da força $\left(\mathrm{F}_{\text {pico }}-\mathrm{F}_{\text {mín }}\right)$ e o tempo para a força pico em cada braçada; expressa em $\mathrm{N}$ por segundo.

7. Impulso $\left(\mathrm{I}_{\mathrm{mp}} \mathrm{F}\right)$ : integral da curva força-tempo entre dois instantes de $\mathrm{F}_{\text {mín }}\left(\mathrm{F}_{\text {mín } 1}\right.$ e $\mathrm{F}_{\text {mín2 }}, \mathrm{F}_{\text {mín2 }}$ e $\mathrm{F}_{\text {mín } 3} \ldots \mathrm{F}_{\text {mín } 8}$ e $\mathrm{F}_{\text {min } 9}$ ), e calculada a partir do método dos trapézios; expresso em N.s.

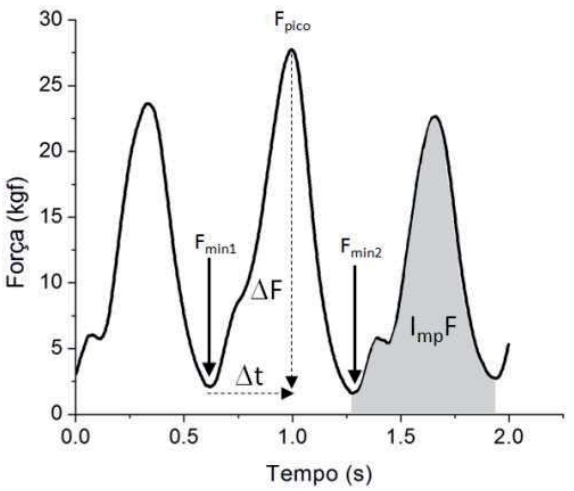

FIGURA 3 - Representação dos pontos de referência utilizados na análise da curva força-tempo.

\section{Análise estatística}

Os dados foram tratados utilizando o pacote estatístico SPSS for Windows (Versão 16.0; SPSS, Inc., Chicago, IL). Para análise foi considerada a média dos parâmetros biomecânicos obtidos nas oito braçadas em cada tentativa. Média e desvio padrão foram utilizados como medida de tendência central e dispersão, respectivamente. A normalidade e a homogeneidade dos dados foram verificadas pelos testes de Shapiro-Wilk e Levene, respectivamente.

Os parâmetros obtidos na primeira e segunda repetição foram comparados pelo teste $t$ para amostras pareadas. A reprodutibilidade relativa, que define “(...) o grau em que cada indivíduo mantém sua posição em uma amostra de medidas repetidas (...)" (MoreIRA et al., 2008, p.416), foi medida pelo coeficiente de correlação de Pearson. A consistência entre as duas tentativas foi testada pelo coeficiente de correlação intraclasse (CCI), obtido pelos resultados da análise de variância de medidas repetidas e classificado da seguinte forma: 0,90-0,99 = alta; $0,80-0,89$ = boa; $0,70-0,79=$ fraca $<0,69$ = pobre, conforme classificação utilizada por Ross, LANGFORD e WHELAN (2002).

A reprodutibilidade absoluta define o grau em que as medidas repetidas variam por indivíduo (MOREIRA et al., 2008) e foi verificada pelo coeficiente de variação $(\mathrm{CV})$, obtido pela razão entre o desvio padrão e a média multiplicada por $100(\mathrm{CV}=$ desvio padrão / média $\mathrm{x}$ 100). Esse procedimento foi adotado para cada caso para que, então, fosse calculado o CV de todo o grupo para cada parâmetro. Em todas as situações o nível de significância adotado foi de $\mathrm{p}<0,05$.

\section{Resultados}

Os valores descritivos dos parâmetros obtidos em cada uma das repetiçôes estão apresentados na TABELA 1.
A TABELA 2 apresenta os valores da diferença pareada entre as duas tentativas para cada parâmetro. Nenhuma diferença significante foi detectada entre 
as duas tentativas para nenhum dos parâmetros analisados.

Os resultados obtidos para a reprodutibilidade relativa $(\mathrm{r}=$ coeficiente de correlação) e absoluta $(\mathrm{CV}=$ coeficiente de variação), e consistência entre as duas repetições $(\mathrm{CCI}=$ coeficiente de correlação intraclasse) estão disponíveis na TABELA 3.
Com exceção da força média, em que a reprodutibilidade foi considerada boa [0,89$0,80]$, todos os demais parâmetros apresentaram reprodutibilidade alta $[0,90-0,99]$. No tocante ao coeficiente de variação, apenas a força mínima apresentou valor superior a $4 \%$.

TABELA 1 - Média e desvio padrão (DP) da força pico $\left(\mathrm{F}_{\text {pico }}\right)$, força média $\left(\mathrm{F}_{\text {méd }}\right)$, impulso $\left(\mathrm{I}_{\mathrm{mp}} \mathrm{F}\right)$, taxa de desenvolvimento de força (TDF), duração da braçada (DUR), tempo para força pico $\left(\mathrm{TF}_{\text {pico }}\right)$ e força mínima $\left(\mathrm{F}_{\text {mín }}\right)$ nas tentativas 1 e 2 .

\begin{tabular}{lcccc}
\hline & Tentativa 1 & \multicolumn{3}{c}{ Tentativa 2 } \\
\cline { 2 - 5 } & Média & DP & Média & DP \\
\hline $\mathrm{F}_{\text {pico }}(\mathrm{N})$ & 272,9 & 26,5 & 273,9 & 28,1 \\
$\mathrm{~F}_{\text {méd }}(\mathrm{N})$ & 146,4 & 10,9 & 147,0 & 10,8 \\
$\mathrm{I}_{\text {mp }} \mathrm{F}(\mathrm{N} \cdot \mathrm{s})$ & 96,5 & 14,3 & 96,3 & 13,3 \\
$\mathrm{TDF}(\mathrm{N} / \mathrm{s})$ & 684,5 & 89,7 & 683,2 & 87,7 \\
$\mathrm{DUR}(\mathrm{ms})$ & 659 & 65 & 656 & 68 \\
$\mathrm{TF}$ & $37 \mathrm{~ms})$ & 37 & 333 & 42 \\
$\mathrm{~F}_{\text {mín }}(\mathrm{N})$ & 332 & 14,4 & 50,7 & 15,7 \\
\hline
\end{tabular}

TABELA 2 - Diferença pareada (dif) entre as duas tentativas para a força pico $\left(\mathrm{F}_{\text {pioo }}\right)$, força média $\left(\mathrm{F}_{\text {méd }}\right)$, impulso $\left(I_{m p} F\right)$, taxa de desenvolvimento de força (TDF), duração da braçada (DUR), tempo para força pico $\left(\mathrm{TF}_{\text {pioo }}\right)$ e força mínima $\left(\mathrm{F}_{\text {mín }}\right)$.

\begin{tabular}{lccccccc}
\hline & Média & DP & EPM & IC 95\% LI & IC 95\% LS & T & P \\
\hline dif $\mathrm{F}_{\text {pico }}$ & $-0,94$ & 9,20 & 2,30 & $-5,84$ & 3,97 & $-0,41$ & 0,69 \\
dif $\mathrm{F}_{\text {méd }}$ & $-0,55$ & 7,84 & 1,96 & $-4,73$ & 3,63 & $-0,28$ & 0,78 \\
dif $\mathrm{I}_{\text {mp }} \mathrm{F}$ & 0,21 & 3,26 & 0,82 & $-1,53$ & 1,95 & 0,26 & 0,80 \\
dif TDF & 1,29 & 45,01 & 11,25 & $-22,69$ & 25,28 & 0,12 & 0,91 \\
dif DUR & 2,99 & 26,40 & 6,60 & $-11,07$ & 17,06 & 0,45 & 0,66 \\
dif TF $_{\text {pico }}$ & $-0,47$ & 22,88 & 5,72 & $-12,66$ & 11,72 & $-0,08$ & 0,94 \\
dif $\mathrm{F}_{\text {mín }}$ & $-0,81$ & 8,14 & 2,03 & $-5,15$ & 3,53 & $-0,40$ & 0,70 \\
\hline
\end{tabular}

$\mathrm{DP}=$ desvio padrão; EPM = erro padrão de medida da diferença; IC 95\% LI = intervalo de confiança $95 \%$ limite inferior;

IC $95 \%$ LS = intervalo de confiança 95\% limite superior;

$\mathrm{t}=$ resultado da estatística t;

$\mathrm{P}=$ probabilidade de significância.

TABELA 3 - Matriz de correlação (r), coeficiente de correlação intraclasse (CCI), e coeficiente de variação (CV) para força pico $\left(\mathrm{F}_{\text {pioo }}\right)$, força média $\left(\mathrm{F}_{\text {méd }}\right)$, impulso $\left(\mathrm{I}_{\mathrm{mp}} \mathrm{F}\right)$, taxa de desenvolvimento de força (TDF), duração da braçada (DUR), tempo para força pico $\left(\mathrm{TF}_{\text {pioo }}\right)$ e força mínima $\left(\mathrm{F}_{\text {mín }}\right)$ nas tentativas 1 e 2.

\begin{tabular}{lccc}
\hline & $\mathbf{r}$ & $\mathbf{C C I}$ & $\mathbf{C V}$ \\
\hline $\mathrm{F}_{\text {pico }}(\mathrm{N})$ & $0,95^{*}$ & 0,97 & $1,7 \%$ \\
$\mathrm{~F}_{\text {méd }}(\mathrm{N})$ & $0,74^{*}$ & 0,85 & $3,3 \%$ \\
$\mathrm{I}_{\text {mp }} \mathrm{F}(\mathrm{N} \cdot \mathrm{s})$ & $0,97^{*}$ & 0,99 & $1,8 \%$ \\
$\mathrm{TDF}(\mathrm{N} / \mathrm{s})$ & $0,87^{*}$ & 0,93 & $3,6 \%$ \\
$\mathrm{DUR}(\mathrm{ms})$ & $0,92^{*}$ & 0,96 & $2,3 \%$ \\
$\mathrm{TF}_{\text {pico }}(\mathrm{ms})$ & $0,84^{*}$ & 0,91 & $3,7 \%$ \\
$\mathrm{~F}_{\text {min }}(\mathrm{N})$ & $0,86^{*}$ & 0,92 & $10,3 \%$ \\
\hline
\end{tabular}

${ }^{*} p<0,001$. 


\section{Discussão}

O objetivo do presente estudo foi determinar a reprodutibilidade dos parâmetros da curva forçatempo do estilo "Crawl" em um protocolo de curta duração no nado atado. Para isso foram calculados os coeficientes de correlação intraclasse (CCI) e de variação $(\mathrm{CV})$ e também a matriz de correlação e o erro padrão de medida (EPM) para duas tentativas.

A reprodutibilidade relativa foi verificada pelo coeficiente de correlação de Pearson e apresentou resultados satisfatórios ao variar entre 0,95 e 0,74 $(\mathrm{p}<0,001)$, dependendo do parâmetro analisado. Estes resultados foram semelhantes aos apresentados em investigações prévias, que verificaram correlação entre teste e re-teste de 0,96 para a força pico (Marinho \& ANdries Júnior, 2001) e 0,93 para o impulso (PАРОтI et al., 2003) quando avaliados em protocolos de 10 e $30 \mathrm{~s}$, respectivamente.

No tocante à consistência entre as duas tentativas, foi observada alta reprodutibilidade para força pico, impulso, taxa de desenvolvimento de força, duração da braçada, tempo para força pico e força mínima, que apresentaram CCI acima de 0,90, e boa reprodutibilidade para a força média, em que o CCI ficou entre 0,80 e 0,89 . Essa classificação do CCI se baseou na proposta de Ross, LANGFORD e WHELAN (2002) (0,90-0,99 = alta; 0,80-0,89 = boa; $0,70-0,79=$ fraca; $<0,69=$ pobre) $\mathrm{e}$, embora não seja consensual, tem sido empregada em estudos recentes (Moreira, Okano, RonQue, Souza, \& Oliveira, 2006; Moreira et al., 2008).

A reprodutibilidade absoluta, que segundo ATKINSON e NeVIL (1998) tem sido considerada aceitável quando o coeficiente de variação se situa abaixo de $10 \%$, apesar de este valor ainda ser mérito de conjectura (Moreira et al., 2008), também foi alvo de análise no presente estudo. Neste sentido, apenas a força mínima não atendeu a este "valor de corte" (10,3\%). Em uma perspectiva prática, esses achados sugerem que este parâmetro biomecânico, gerado a partir da análise dos esforços de $10 \mathrm{~s}$ no nado atado, deve ser visto com cautela. De todos os parâmetros analisados, a força mínima parece ser a variável mais suscetível a variação de tentativa para tentativa, fato que, por exemplo, dificulta o uso desta medida para monitoramento do processo de preparação. Por outro lado, nos demais parâmetros, o coeficiente de variação, que oscilou entre 1,7 e 3,7\%, demonstra a confiabilidade destas medidas, conferindo robustez para a utilização destes parâmetros em uma perspectiva prática e teórica.

O EPM sugere uma amplitude dentro da qual um "escore verdadeiro" de um sujeito pode ser esperado quando considerado o erro associado às medidas repetidas (Ross, LANGFord \& Whelan, 2002). Por exemplo, com um EPM de 2,3 N, se um indivíduo alcança uma força pico de $300 \mathrm{~N}$ no teste de nado atado, pode-se afirmar, com $95 \%$ de confiança, que o escore verdadeiro para este indivíduo estaria entre 304,6 e 295,4 N ( \pm 2 EPM). Deste modo, uma alteração superior a $4,6 \mathrm{~N}$ no escore deste sujeito poderia representar uma alteração real em seu desempenho que não está associada a um erro de medida. $\mathrm{O}$ mesmo raciocínio deve ser empregado para o EPM dos demais parâmetros analisados. Esta abordagem tem ampla aplicação no controle das respostas dos esportistas ao processo de treinamento, pois possibilita a identificação do real incremento ou diminuição do desempenho, reduzindo as chances de uma avaliação equivocada sobre as respostas dos atletas ao longo da preparação, nos diferentes momentos de coleta.

É razoável admitir que indivíduos altamente treinados e com ampla familiarização ao teste proposto tendem a apresentar uma maior consistência em medidas repetidas do que indivíduos não-atletas. A não-significância do teste $\mathrm{t}$ para as duas tentativas indica que fatores como o efeito de aprendizagem, motivação ou inconsistência de protocolo não influenciaram a avaliação. Esse fato não exclui a possibilidade de ocorrência de variação nos resultados (CCI, EPM, $\mathrm{r}$, entre outros) caso três ou mais esforços fossem considerados. Contudo, em face dos dados apresentados, acreditamos que esta seria mínima e não-significante.

Assim, o alto coeficiente de correlação, alto CCI, baixo CV e EPM, e ainda a ausência de significância estatística entre as diferenças das duas tentativas para os parâmetros biomecânicos analisados indicam a reprodutibilidade do protocolo de $10 \mathrm{~s}$ no nado atado para nadadores competitivos em nível nacional. Apesar disso, é preciso identificar em futuras investigações quais destes parâmetros possuem maior sensibilidade às alterações no desempenho.

No tocante às limitações, é importante considerar que o presente estudo analisou somente a consistência interna (reprodutibilidade teste-reteste intra-dia) dos parâmetros biomecânicos obtidos no nado atado. Questôes acerca da estabilidade (reprodutibilidade teste-reteste inter-dias) não foram avaliadas e podem ser alvo de investigações futuras.

Ademais, ressaltamos que o total de oito braçadas analisadas compreendeu aproximadamente $5,5 \mathrm{~s}$ (duração média da braçada x número de braçadas) e foi adotado em função dos autores o considerarem 
representativo para 10 s. Todavia, em protocolos com duração superior a esta será necessário analisar um número maior, de modo a abranger um maior percentual da duração total do teste.

\title{
Conclusão
}

A partir das informaçōes obtidas é possível concluir que os parâmetros biomecânicos analisados a partir da curva força-tempo no nado atado apresentam alta reprodutibilidade em protocolo de curta duração, possibilitando, portanto, o monitoramento das possíveis alterações dos parâmetros de força ao longo do processo de treinamento. Adicionalmente, os achados do presente estudo revelaram que a força mínima deve ser analisada com cautela devido ao seu coeficiente de variação elevado.

\begin{abstract}
Reliability of front-Crawl's force-time curve in a short duration protocol

The aim of the present study was to analyze the reliability of biomechanical parameters of the front-Crawl's force-time curve in a 10-s protocol. Sixteen national competitive male swimmers $(20.4 \pm 4.0$ years; $100-m$ best time: $53.68 \pm 0.99 \mathrm{~s}$ ) performed two $10-\mathrm{s}$ maximal efforts in tethered swimming. Peak force, average force, impulse, rate of force development, stroke duration, time to peak force and minimum force were represented by the mean of eight consecutive strokes obtained in each trial. The paired student-t test was used to verify differences between trials for each biomechanical parameter. The significance level adopted was 5\%. Relative reliability was tested by Pearson correlation coefficient and the consistency between trials by means of intraclass coefficient (CCI). The coefficient of variation was used to verify the absolute reliability. No significant differences between the trials to any biomechanical parameter were found. High $\mathrm{CCl}$ values and low $\mathrm{CV}$ values revealed the high internal consistency of the analyzed variables. It can be concluded that the biomechanical parameters regarding the tethered swimming are reliable in short duration protocols and may be used by coaches and athletes for monitoring training.
\end{abstract}

UnITERMS: Reliability; Swimming; Pulling force; Tethered swimming.

\section{Referências}

ATKINSON, G.; NEVILL, A.M. Statistical methods for assessing measurement error (reliability) in variables relevant to sports. Sports Medicine, Auckland, v.26, p.217-38, 1998.

ADAMS, T.A.; MARTIN, R.B.; YEATER, R.A.; GILSON, K.A. Tethered force and velocity relationships. Swimming

Technique, Sedona, v.20, p.21-6, 1983.

BARBOSA, A.C.; DOPSAJ, M.; OKICIC, T.; ANDRIES JUNIOR, O. The usefulness of the fully tethered swimming for 50-m breaststroke performance prediction. In: KJENDLIE, P.L; STALLMAN, R.K.; CABRI, J. (Orgs.). Biomechanics and medicine in swimming XI. Oslo: Nordbergtrykk, 2010. p.47-9.

BOLLENS, E.; ANNEMANS, L.; VAES, W.; CLARYS, J.P. Peripheral EMG comparison between fully tethered and free front crawl swimming. In: UNGERECHTS, B.E.; REISCHLE, K.; WILKE, K. (Orgs.). Swimming science V. Champaign: Human Kinetics, 1988. p.173-81.

BONEN, B.; WILSON, A.; YARKONY, M.; BELCASTRO, A.N. Maximal oxygen uptake during free, tethered, and flume swimming. Journal of Applied Physiology, Bethesda, v.48, n.2, p.232-5, 1980.

CABRI, J.; ANNEMANS, 1.; CLARYS, J.P.; BOLLENS E.; PUBLIE, J. The relation of stroke frequency, force, and EMG in front crawl tethered swimming. In: UNGERECHTS, B.E.; REISCHLE, K.; WILKE, K. (Orgs.). Swimming science V. Champaign: Human Kinetics, 1988. p.183-9. 
CASTRO, F.A.S.; OLIVEIRA, T.S.; MORÉ, F.C.; MOTA, C.B. Relações entre desempenho em 200 m nado crawl e variáveis cinéticas do teste de nado estacionário. Revista Brasileira de Ciências do Esporte, Campinas, v.31, n.3, p.161-76, 2010. DOPSAJ, M.; MATKOVIC, I.; THANOPOULOS, V.; OKICIC, A. Reliability and validity of basic kinematics and mechanical characteristics of pulling force in swimmers measured by method of tethered swimming with maximum intensity of 60 seconds. Facta Universitatis - Series: Physical Education and Sport, Nis, v.1, n.10, p.11-22, 2003

DOPSAJ, M.; MATKOVIC, I.; ZDRAVKOVIC, I. The relationship between 50m-freestyle results and characteristics of tethered forces in male sprint swimmers: a new approach to tethered swimming test. Facta Universitatis - Series: Physical Education and Sport, Nis, v.1, n.7, p.15-22, 2000.

GLAISTER, M. Multiple sprint work: physiological responses, mechanisms of fatigue and the influence of aerobic fitness. Sports Medicine, Auckland, v.35, n.9, p.757-7, 2005.

HOLMÉR, I. Physiology of swimming man. Exercise and Sports Science Review, Philadelphia, v.7, p.87-123, 1979. MAGLISCHO, C.W.; MAGLISCHO, E.W.; SHARP, R.L.; ZIER, D.J.; KATZ, A. Tethered and non-tethered crawl swimming. In: TERAUDS, J.; BARTHELS, K.; KREIGHBAUM, E.; MANN, R.; CRAKES, J. (Orgs.). Proceedings of ISBS: sports biomechanics. Del Mar: Academic Publisher, 1984. p.163-76.

MARINHO P.C.; ANDRIES JÚNIOR, O. Avaliação da força propulsora do nadador: validação e reprodutibilidade de uma metodologia específica. Revista Brasileira de Ciência e Movimento, p.79, 2001. Suplemento.

MARINHO, P.C.; BERNHOEFT, M.; MOURA, R.; CIPOLLI, E.; ANDRIES JÚNIOR, O. Análise comparativa da força especial de nadadores de alto rendimento especializados nas distâncias de 100 e 200 metros crawl. In: CONGRESSO DE CIÊNCIAS DO DESPORTO, 1., 2005, Campinas. Anais.... Campinas: FEF/Unicamp, 2005. Disponível em: <www. fef.unicamp.br/hotsites/ccd_2005/.../Paulo\%20Cezar\%20Marinho.pdf>.

MARINHO, P.C.S. Nado amarrado: mensuração da força propulsora e sua relação com a velocidade básica de nadadores de nível competitivo. 2002. Dissertação (Mestrado) - Universidade Estadual de Campinas, Campinas, 2002.

MARINHO, P.C.S.; GOMES, A.C. Diagnóstico dos níveis de força especial em nadadores e sua influência no resultado desportivo. Treinamento Desportivo, Londrina, v.4, n.2, p.41-7, 1999.

MOREIRA, A.; MAIA, G.; LIZANA, C.R.; MARTINS, E.A.; OLIVEIRA, P.R. Reprodutibilidade e concordância do teste de salto vertical com contramovimento em futebolistas de elite da categoria sub-21. Revista da Educação Física, Maringá, v.19, n.3, p.413-21, 2008.

MOREIRA, A.; OKANO, A.H.; RONQUE, E.V.; SOUZA, M.; OLIVEIRA, P.R. Reprodutibilidade dos testes de salto vertical e salto horizontal triplo consecutivo em diferentes etapas da preparação de basquetebolistas de alto rendimento.

Revista Brasileira de Cineantropometria e Desempenho Humano, Florianópolis, v.8, n.4, p.66-72, 2006.

MOROUÇO, P.; KESKINEN, K.L;, VILAS-BOAS, J.P.; FERNANDES, R.J. Relationship between tethered forces and the four swimming techniques performance. Journal of Applied Biomechanics, Champaign, v.27, n.2, p.161-9, 2011. PAPOTI, M.; MARTINS, L.; CUNHA, S.; ZAGATTO, A.; GOBATTO, C. Padronização de um protocolo específico para determinação de aptidão anaeróbia de nadadores utilizando células de carga. Revista Portuguesa de Ciências do Desporto, Porto, v.3, n.3, p.36-42, 2003.

PAPOTI, M.; MARTINS, L.E.B.; CUNHA, S.A.; ZAGATTO, A.M.; GOBATTO, C.A. Effects of taper on swimming force and swimmers performance after an experimental ten-week training program. Journal of Strength and Conditioning Research, Champaign, v.21, n.2, p.538-42, 2007.

PESSÔA-FILHO, D.M.; NASCIMENTO, E.P.; DENADAI, B.S. Validação do modelo potência-tempo limite no crawlatado pelas relações com os modelos distância/tempo limite e velocidade/tempo limite. Revista Brasileira de Ciências do Esporte, Campinas, v.29, n.2, p.95-114, 2008.

ROSS, M.D.; LANGFORD, B.; WHELAN, P.J. Test-retest reliability of 4 single-leg horizontal hop tests. Journal of Strength and Conditioning Research, Champaign, v.16, n.4, p.617-22, 2002.

RISCH, O.; CASTRO, F. A. S. Desempenho em natação e pico de força em tethered swimming. In: CONGRESSO BRASILEIRO DE BIOMECÂNICA, 12., 2007, São Pedro. Anais... São Pedro: Tec Art, 2007. p.441-6.

SZMUCHROWSKI, L.A.; FERREIRA, R.M.; CARVALHO, R.G.S.; FERREIRA, J.C. Correlação entre a força isométrica, força de propulsão de nado e velocidade média em nadadores. In: CONGRESSO BRASILEIRO DE BIOMECÂNICA, 12., 2007, São Pedro. Anais... São Pedro: Tec Art, 2007. p. 620-5.

WINTER, D.A. Biomechanics and motor control of human movement. Toronto: Wiley Interscience, 1990.

YEATER, R.A.; MARTIN, R.B.; WHITE, M.K.; GILSON, K.H. Tethered swimming forces in the crawl, breast and back strokes and their relationship to competitive performance. Journal of Biomechanics, Philadelphia, v.14, n.8, p.527-37, 1981.

44 • Rev. bras. Educ. Fís. Esporte, São Paulo, v.26, n.1, p.37-45, jan./mar. 2012 


\section{Agradecimentos}

Os autores agradecem a CAPES pelo suporte financeiro e ao CEFISE pelo nado atado.

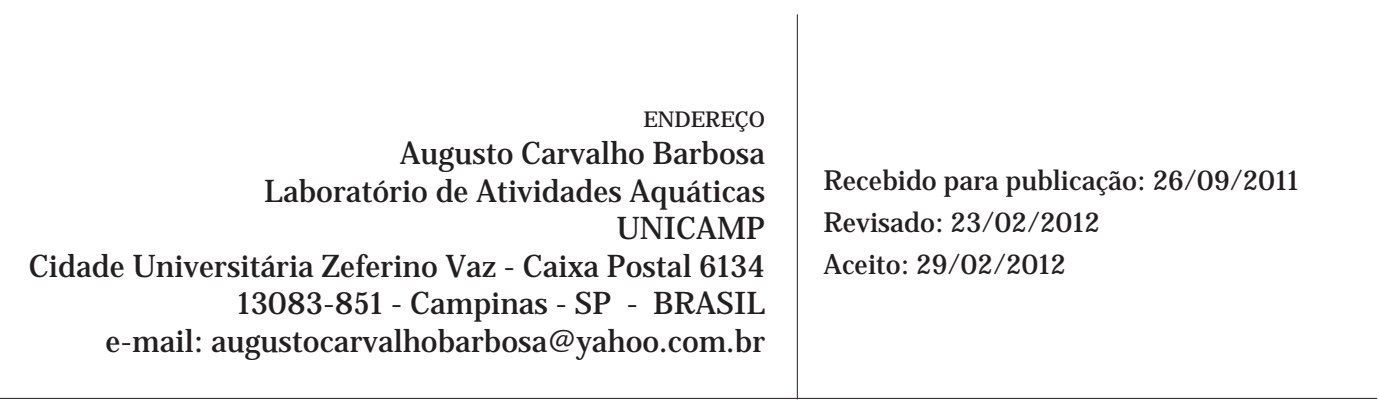

Rev. bras. Educ. Fís. Esporte, São Paulo, v.26, n.1, p.37-45, jan./mar. 2012 • 45 